\title{
Erratum
}

\section{The Ground State Energy of a 2-D Electron Gas}

A. Isihara and T. Toyoda

Z. Physik B 26, 216 (1977)

The correlation energy should read as

$\varepsilon_{c}=-0.6258 \pm 0.002$

$+(1.074 \pm 0.02) r_{s}-0.1728 r_{s} \ln r_{s}$

A. Isihara

Department of Physics

Statistical Physics Laboratory

State University of New York

at Buffalo

Buffalo, New York 14260

USA 